\title{
Volumetric Pattern Analysis of Airborne Antennas
}

\author{
C. L. YU, MEMBER, IEEE, W. D. BURNSIDE, MEMBER, IEEE, AND M. C. GILREATH
}

\begin{abstract}
By blending together the roll and elevation plane highfrequency solutions, a very efficient technique has been developed for the volumetric pattern analysis of antennas mounted on the fuselage of a generalized aircraft. The fuselage is simulated by an infinitely long, perfectly conducting, elliptic cylinder in cross section and a composite elliptic cylinder in profile. The wings, nose section, stabilizers, and landing-gear doors may be modeled by finite flat or bent plates. Good agreement with accurate scale model measurements has been obtained for a variety of airborne antenna problems.
\end{abstract}

\section{INTRODUCTION}

I F MODERN aircraft antennas are to function properly, the antenna pattern must meet certain system requirements. The conventional design procedure for an antenna on a particular aircraft has been to evaluate the performance of a candidate antenna system based on numerous scale-model measurements. This approach requires a great deal of engineering time and expense. The numerical technique presented in this paper alleviates the problems associated with measurements for fuselage-mounted antennas by providing an efficient means of accurately calculating volumetric patterns.

Since the majority of airborne antennas operate above 100 $\mathrm{MHz}$, a high-frequency approach is appropriate. As a result, the geometrical theory of diffraction (GTD) is applied to develop this numerical solution. Before discussing the actual aircraft solutions, it will be helpful to consider two basic GTD structures, the wedge and the curved surface. For the wedge problem, the total field is defined as the superposition of the incident, reflected, and diffracted fields. The wedge diffracted field component is related to the geometry and field incident on the edge as given in [1]. In the case of the curved-surface problem, the radiated field is described in terms of rays which propagate outward from the antenna to the field point as presented in [2]. In the illuminated region, the dominant ray trajectory is a straight line joining the antenna and field point; whereas in the shadow region, the energy propagates around the curved surface along a geodesic path from which it diffracts tangentially toward the field point. Since the total field for antennas mounted on or near these structures can be written in terms of a few dominant rays, one can simulate complex structures using a combination of these basic geometries. Using this concept in the conventional way, one must trace a ray (or energy flow) as it is scattered between the various components simulating the structure. Even though this approach is very powerful, it can become quite cumbersome if too many higher

Manuscript received March 14, 1977; revised December 5, 1977. This work was supported in part by Grant Number NGL 36-008-138 between the National Aeronautics and Space Administration and The Ohio State University Research Foundation.

C. L. Yu is with the Naval Weapons Center, China Lake, CA 93555.

W. D. Burnside is with the ElectroScience Laboratory, Department of Electrical Engineering, The Ohio State University, Columbus, $\mathrm{OH}$ 43212 .

M. C. Gilreath is with the National Aeronautics and Space Administration, Langley Research Center, Hampton, VA 23665. order interactions need to be taken into account. The analysis presented in this paper illustrates how the GTD solution approach can be successfully applied to solve a complex electromagnetic problem by appropriately including just a few dominant terms.

Before describing the refinements developed in this paper, let us consider the mechanics of the GTD approach as it has been previously applied to aircraft antenna problems. First, the aircraft structure is broken up into simpler parts in such a way that these parts simulate the essential character associated with the aircraft's scattered fields. These parts are individually analyzed and later combined to simulate the complete aircraft. In the roll plane pattern solution of [3], the wings were treated as finite flat plates. Therein, it was shown that the GTD could successfully predict the total field for an antenna in the presence of a finite flat plate simply by superimposing the incident, reflected, and various edge diffracted fields. A more general roll plane model was used in [4], where the flat plate wing and elliptic cylinder fuselage models were combined using the GTD approach. The dominant rays included in that solution are illustrated in Fig. 1. Note that in this case the wing is illuminated by energy which flows around the fuselage. This modified illumination was analyzed in terms of the geodesic paths by which energy propagates from the antenna around the cylinder, diffracts tangentially from the curved surface, and strikes various portions of the wing. The accuracy of this model was verified in [4].

An elevation plane analysis was also presented in [4], in which the fuselage profile was simulated by a composite elliptic cylinder. This model was not satisfactory in that the nose section and vertical stabilizer were neglected; thus, good agreement with measurements was not obtained in the fore and aft directions. The correction of this shortcoming and the development of a volumetric pattern solution are presented in this paper.

\section{FUSELAGE SIMULATION}

Using the GTD solution approach, let us consider the radiation properties of antennas mounted on fuselage-shaped structures, neglecting the wings, stabilizers, etc. Since the complete volumetric pattern is desired, the three-dimensional nature of the fuselage must be taken into account. Thus a single infinite cylinder model, such as applied in [3], [4], cannot accurately simulate a fuselage for all possible radiation angles, especially those near the cylinder axis. To overcome this limitation, a complete analysis was developed in [5] for antennas mounted on surfaces of revolution. Using this model, the three-dimensional nature of the fuselage was accurately taken into account. Even though this solution provided the desired properties, it is a very complex formulation in that the geodesic paths had to be found by means of a numerical solution. A stored set of geodesic test curves was generated from which one could predict the proper geodesic paths which 


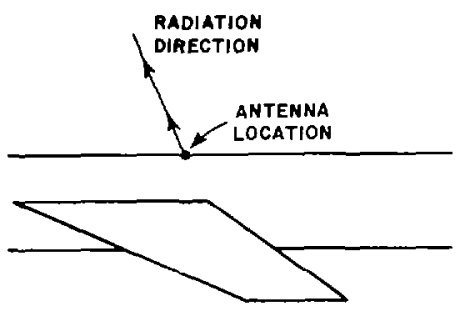

(a) DIRECT TERM ILLUSTRATED

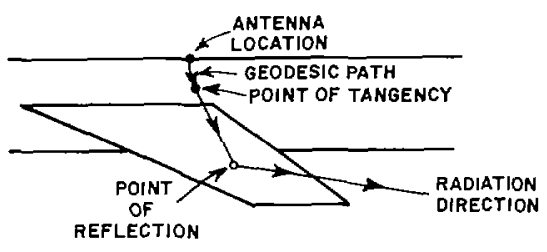

(b) REFLECTED TERM ILLUSTRATED

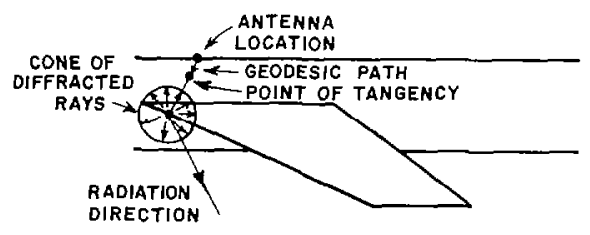

(C) EDGE DIFFRACTED TERM ILLUSTRATED

Fig. 1. Dominant rays used in radiation pattern computation.

diffract tangentially in the desired radiation direction. However, the storage space and manipulation times associated with that data set were excessive, which made the direct solution for this structure impractical.

Since the surface of revolution model did successfully simulate fuselage structures, its properties were investigated with a view toward determining the dominant geodesic trajectories for radiation directions in the shadow region of the antenna. It was found that for such electrically large structures and antennas mounted away from the poles of the structure (i.e., the poles associated with the surface of revolution), there were only four dominant rays. These four rays are shown in Fig. 2. Note that two rays propagate around the cross section (rays $B$ and $C$ ) and two along the profile (rays $A$ and $D$ ). These four rays play a significant role in certain elevation plane pattern calculations. For example, the elevation plane pattern of an axial slot mounted on a prolate spheroid is shown in Fig. 3(a) using the two-dimensional solution of [4]. Note that this solution only considers rays which propagate along the profile (rays $A$ and $D$ in Fig. 2) and obviously does not predict the back lobe. The prolate spheroid result (using all four rays) for the same configuration is shown in Fig. 3(b). In this case, the calculated and measured results are in good agreement. This indicates that the two rays which propagate around the fuselage also contribute significantly in the elevation plane. Consequently, this effect must be included to properly simulate the radiation properties for antennas mounted on fuselage-shaped structures.

The two rays which propagate around the cross section are quite similar to the ones which propagate around the roll plane model in [4]; whereas, the two rays which propagate along the profile are similar to those in the elevation plane model. This correspondence is depicted in Fig. 2. With this similarity in

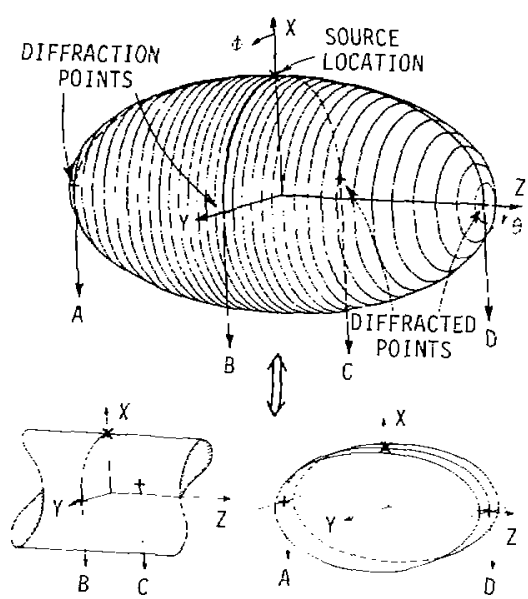

Fig. 2. Four dominant rays creeping around prolate spheroid, plus correspondence using two cylinder solution.

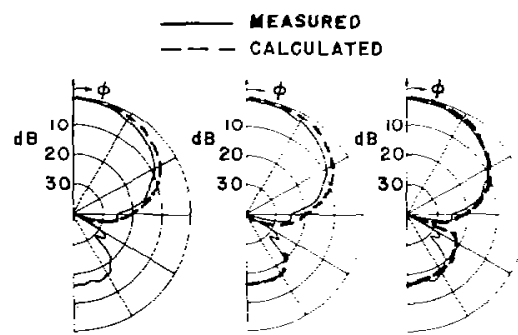

(a)

(b)

(c)

Fig. 3. Elevation plane pattern of axial slot mounted on $4 \lambda$ by $2 \lambda$ prolate spheroid. (a) Two-dimensional solution. (b) Three-dimensional prolate spheroid solution. (c) Present three-dimensional solution.

mind, numerous calculated patterns were compared with measured results for a wide range of radiation directions to ascertain where the individual roll and elevation plane models failed. It was determined that the individual solutions failed, for all practical purposes, in different sectors of the volumetric pattern. Specifically, the roll plane model (an elliptic cylinder whose cross section simulates the fuselage cross section at the antenna location) was found to provide accurate results everywhere except near the fore and aft sectors (i.e., within $10^{\circ}$ to $20^{\circ}$ of the roll plane cylinder axis). On the other hand, these sectors were very adequately analyzed using the elevation fuselage model, which is a composite elliptic cylinder whose cross section simulates the fuselage profile at the antenna location. Consequently, a set of regional solutions was developed which uses either roll or elevation or both models to compute the patterns within a given region. A composite drawing of these regions is shown in Fig. 4. Note that the angle $\alpha$ is chosen such that the solutions tend to blend smoothly together across the regional boundaries. Based on numerous comparisons with scale model measurements, the angle $\alpha$ should be set to a value between $10^{\circ}$ and $20^{\circ}$.

To illustrate the validity of this composite solution, the elevation pattern of an axial slot mounted on a prolate spheroid was calculated and compared with a measured result as shown in Fig. 3(c). It is clear from this comparison that the composite solution does predict the backlobe. The complete validity of this composite fuselage solution will be demonstrated later in terms of various pattern comparisons with scale-model measurements of actual aircraft structures. 


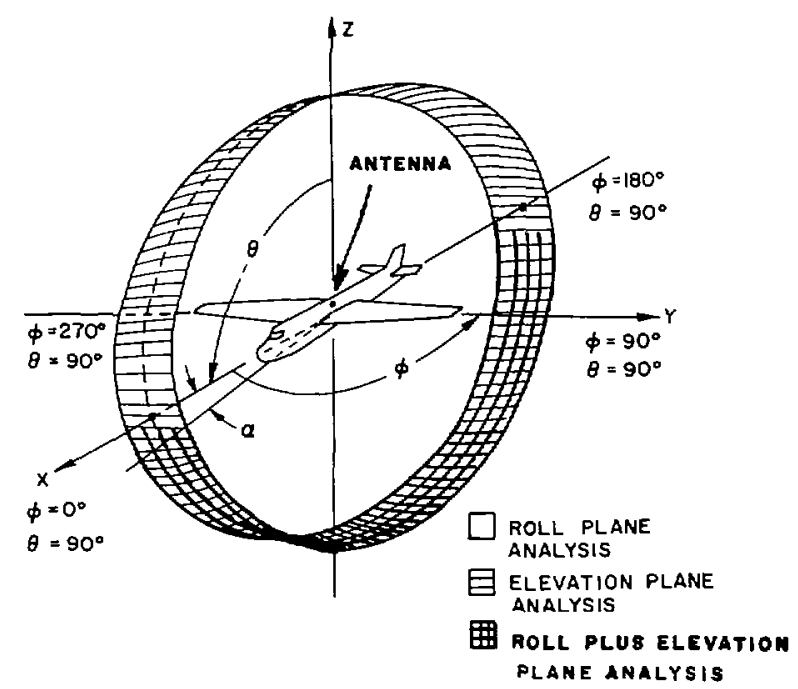

Fig. 4. Illustration of regions used in volumetric pattern solution.

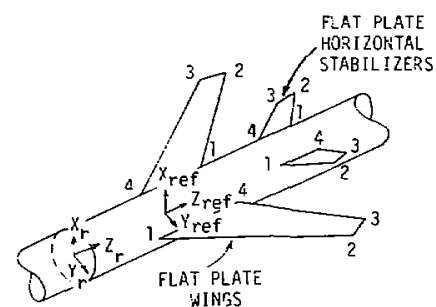

(a)

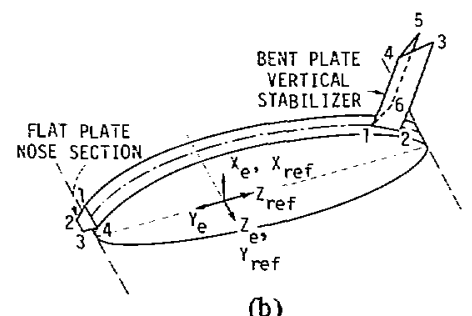

(b)

Fig. 5. (a) Illustration of roll plane model. (b) Illustration of elevation plane model.

\section{AIRCRAFT SIMULATION}

Now that the fuselage may be adequately analyzed in an efficient manner, a simulation model for the complete aircraft can be developed using the GTD solution approach. As demonstrated in [3], [4], the wings and horizontal stabilizers are adequately represented by flat plates. Further, it is shown that these structures are illuminated by energy which flows around the fuselage cross section. Consequently, the wings and horizontal stabilizers must be added to the roll plane model of the fuselage as shown in Fig. 5(a). Note that this model is identical to that analyzed previously in [4]. In terms of the complete volumetric pattern, the scattered fields from the wings and horizontal stabilizers are included in all radiation directions; whereas, the roll plane fuselage fields are only included in the regions indicated in Fig. 4.

The elevation plane model is used to simulate the finite length effects of the fuselage. Since the nose section and vertical stabilizer are illuminated by energy which flows along the fuselage profile, these structures must be added to the elevation plane model. Before the nose section can be adequately simulated, a practical representation for the radome must be found. A comprehensive study of radomes and their effect on the radiation patterns of antennas mounted in their vicinity is far too complex to be considered here. In fact, the analysis of the scattering properties of radomes and the structures mounted under them is an interesting and relevant problem worthy of investigation. For simplicity it is assumed here that the radome is perfectly transparent. This is not an overt assumption in that radomes are designed to be transparent at least at certain frequencies. This leaves a short blunt-looking nose section which extends out from the front of the aircraft. Various complex structures were investigated to simulate this section, all of which led to very inefficient computations. Further, it was found that the nose section normally has little effect on the resulting pattern. Consequently, the nose section for simplicity is simulated by a finite flat plate as shown in Fig. 5(b). This flat plate simply models the major dimensions of the nose section as illustrated in Fig. 6 in terms of a Boeing 737 simulation.

Since many airborne antennas are mounted on or near the fuselage center line, the vertical stabilizer must be simulated

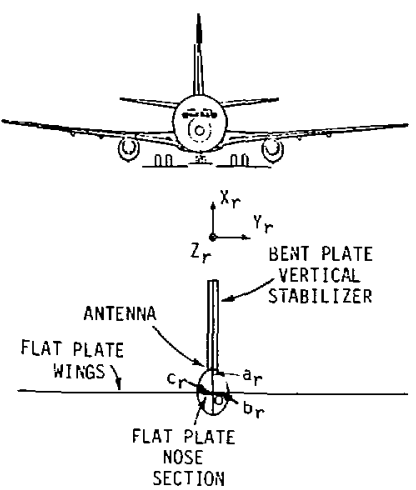

(a) front vien
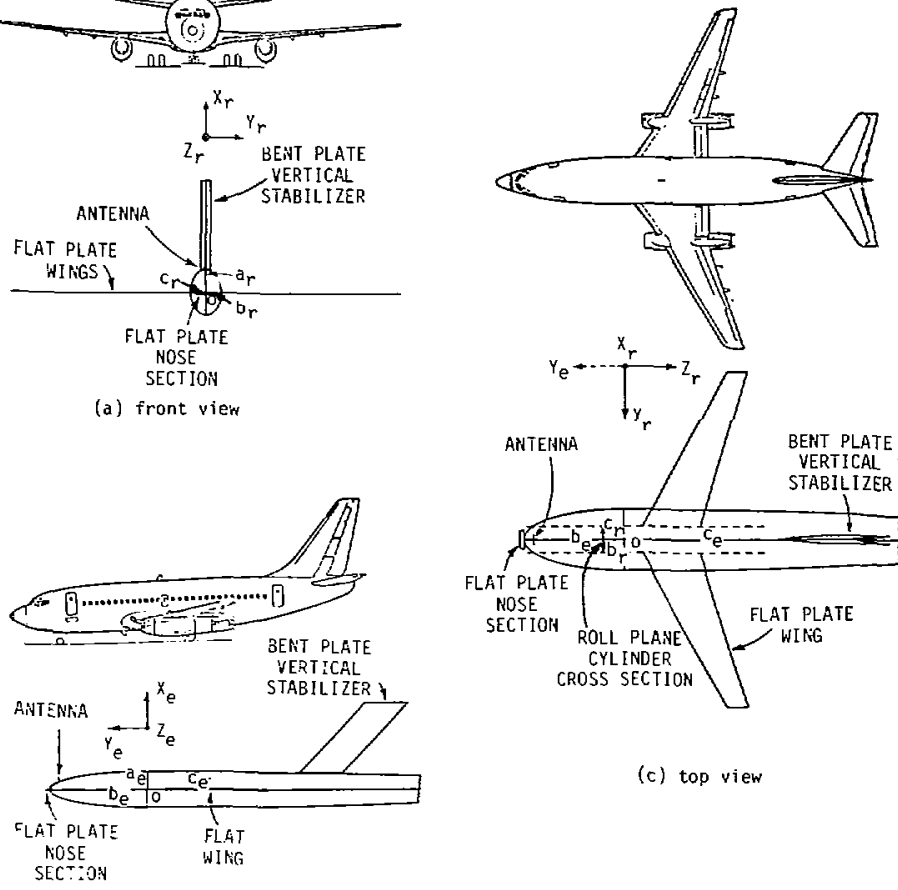

(c) top view

(b) side vien

Fig. 6. Computer simulation model of Boeing 737 aircraft with monopole located at Station 220 on top of fuselage.

by a structure with finite thickness. This thickness is important in that it tends to shadow the direct field from the antenna for aft radiation directions. In order to approximate this effect and maintain the finite flat plate representation of structures that are attached to the fuselage, the vertical stabilizer is modeled by a bent plate as shown in Fig. 5(b). The significant features associated with this simulation are that the leading edge and thickness of bent plate accurately approximate those features of the actual vertical stabilizer. Such a simulation for the Boeing 737 is shown in Fig. 6. Since this 
elevation plane model is again composed of cylinders and plates, its analysis follows that presented in [4]. The validity of this model will be demonstrated in the next section.

To summarize this solution, the fuselage scattered fields are analyzed in terms of the composite approach presented in the previous section. The wings, stabilizers, and nose section are simulated as finite flat or bent plates whose scattered fields are included for all radiation directions. Superimposing all of these scattered field terms is not unduly time consuming in that only a few contribute significantly in a given radiation direction. A complete computer program has been written which takes advantage of this feature and was used to provide the calculated results presented in the next section.

\section{RESULTS}

To illustrate the validity of this numerical solution in predicting the radiation patterns of fuselage-mounted antennas, it is applied to several airborne antenna problems. In each case a calculated pattern is compared with a measured result obtained using an accurate scale model of the antenna and aircraft under investigation.

The elevation plane radiation pattern of a circumferential waveguide mounted on top of a $\mathrm{KC}-135$ aircraft is shown in Fig. 7. The $K_{a}$-band waveguide aperture fields are simulated by an array of 15 infinitesimal elements as discussed in [4]. As shown in Fig. 7, the agreement between the calculated and measured results is very good. Further, the discrepancy displayed in the previous solution [4] in the aft sector no longer exists, since the vertical stabilizer is included in the present simulation. Similar agreement was obtained for the axial waveguide and monopole patterns as presented in [6].

The computer model used to simulate the Boeing 737 aircraft for an antenna mounted at Station 220 above the cockpit is illustrated in Fig. 6. Recall that the ellipses used to simulate the aircraft should model the surface curvature as accurately as possible at the antenna location. Thus the roll model ellipse as shown in the front view (refer to Fig. 6(a)) is a best fit curve to the aircraft cross section at Station 220 and not the major cross section of the aircraft. Various elevation and azimuth radiation patterns for a $\lambda / 4$ monopole mounted on this computer model have been compared with experimental results in [6]. In each case, the calculated results were found to be in very good agreement with measurements. In order to illustrate the overall accuracy, the complete volumetric patterns in terms of the directive gain are presented in Fig. 8. The various directive gain regions are indicated by the color code. For example, the red color indicates the region of space where the gain level is greater than $0 \mathrm{~dB}$. In other words, this is a region where the radiation intensity of the antenna of interest is greater than that of an isotropic point source. The yellow color indicates the region where the radiation intensity is greater than $-3 \mathrm{~dB}$ but less than $0 \mathrm{~dB}$ relative to isotropic. Similarly, the green, blue, purple, dark blue, and gray or black stand for $-6 \mathrm{~dB},-10 \mathrm{~dB},-15 \mathrm{~dB},-20 \mathrm{~dB}$, and less than $-20 \mathrm{~dB}$ levels in gain, respectively. Good agreement is obtained for each of the gain levels. Note that the theta and phi variables used in the pattern plots are defined in Fig. 4.

The experimental results obtained for the 737 aircraft were measured in an RF anechoic chamber using the one-eleventh scale model shown in Fig. 8. An incremental magnetic tape recorder was used to record the pattern data every $2^{\circ}$ in both theta and phi directions. Based on the directivity calculations,

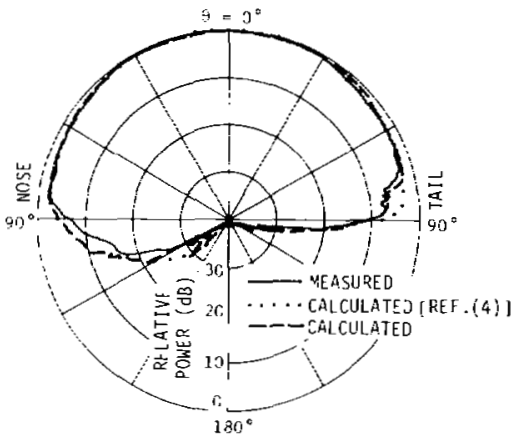

Fig. 7. Elevation plane pattern for circumferential $K_{a}$-band waveguide mounted on $\mathrm{KC}-135$ aircraft.

this increment is sufficient to provide accurate pattern resolution. Experimental data obtained for other antenna locations and types on the Boeing 737 are presented in [7].

To further demonstrate the versatility of this solution, the radiation patterns for the Lindberg crossed-slot antenna mounted at Station 470 along the top center line of a $\mathrm{KC}-135$ aircraft have been analyzed. The Lindberg antenna as discussed in [8] is a UHF antenna designed for use in a satellite-to-aircraft communications link. Using our computer model, the patterns were computed for a right circularly polarized Lindberg antenna. Various calculated patterns along with the measured results as taken from [8] are presented in Fig. 9. In each case, the $E_{\theta}$ pattern corresponds to the vertical component and $E_{\phi}$ to the horizontal component. The gain level in each case is adjusted to compare with measurements. Note that all patterns are computed at a frequency of $250 \mathrm{MHz}$ and $0.78 \lambda$ long slots are considered. Again, good agreement is obtained.

\section{CONCLUSIONS}

The solution developed in this paper combines the roll and elevation plane model analyses to predict the complete volumetric pattern for fuselage-mounted antennas. The significance of this approach is that it provides an efficient and accurate solution to this relevant problem. For example, it can compute a conical pattern in approximately $30 \mathrm{~s}$ on a CDC6600 digital computer. As a result, an antenna designer can use this numerical solution to quickly examine various configurations in order to evaluate various candidate designs and locations based on a set of pattern constraints. This design procedure has been applied to locate and design a microwave landing system antenna for application on the Boeing 737 aircraft. The results of this study have been presented in [6] .

This analysis is based on the geometrical theory of diffraction solution approach which is a high-frequency technique. The lower frequency limit of its application is approximately $100 \mathrm{MHz}$, which is dictated by the requirement that the width of the wing tip be at least a quarter wavelength. The upper frequency limit is dependent on how well the numerical solution model simulates the dominant scattering mechanisms of the actual aircraft. It has been successfully applied for frequencies as high as $35 \mathrm{GHz}$. One other limitation of the analysis is that the antenna and various scattering centers must be separated by at least a wavelength. This implies that the antenna cannot be located near an attached plate. If the previous constraints are satisfied, this solution should provide accuracies at least equivalent to those obtained using scale model measurements. 


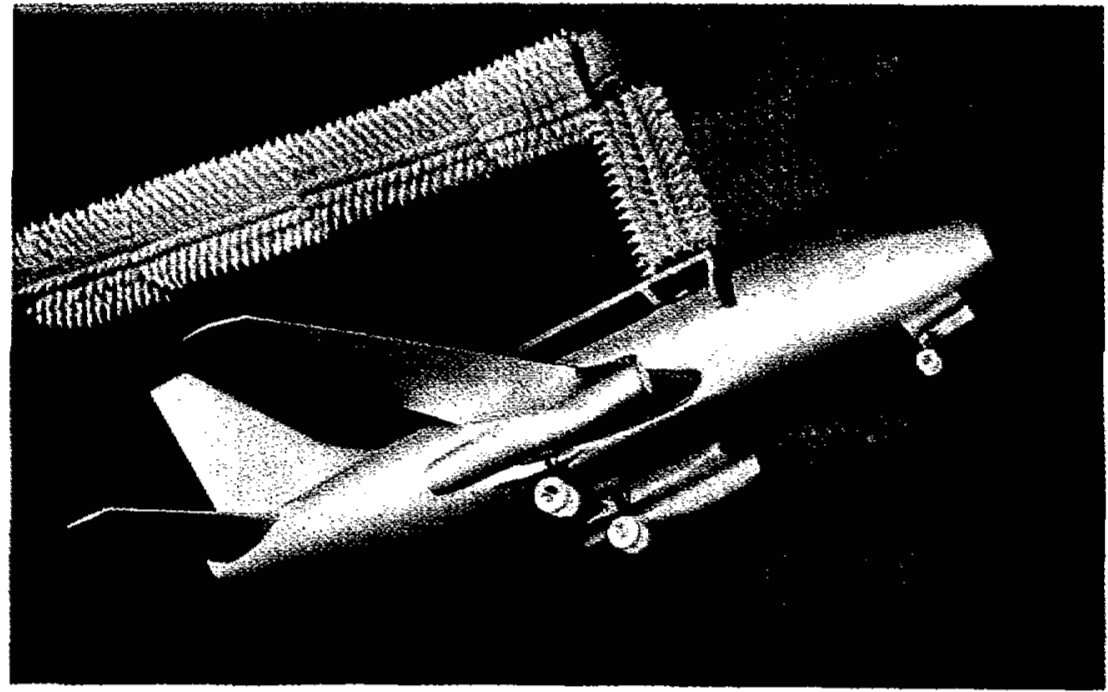

(a) One-eleventh scale model of a Boeing 737

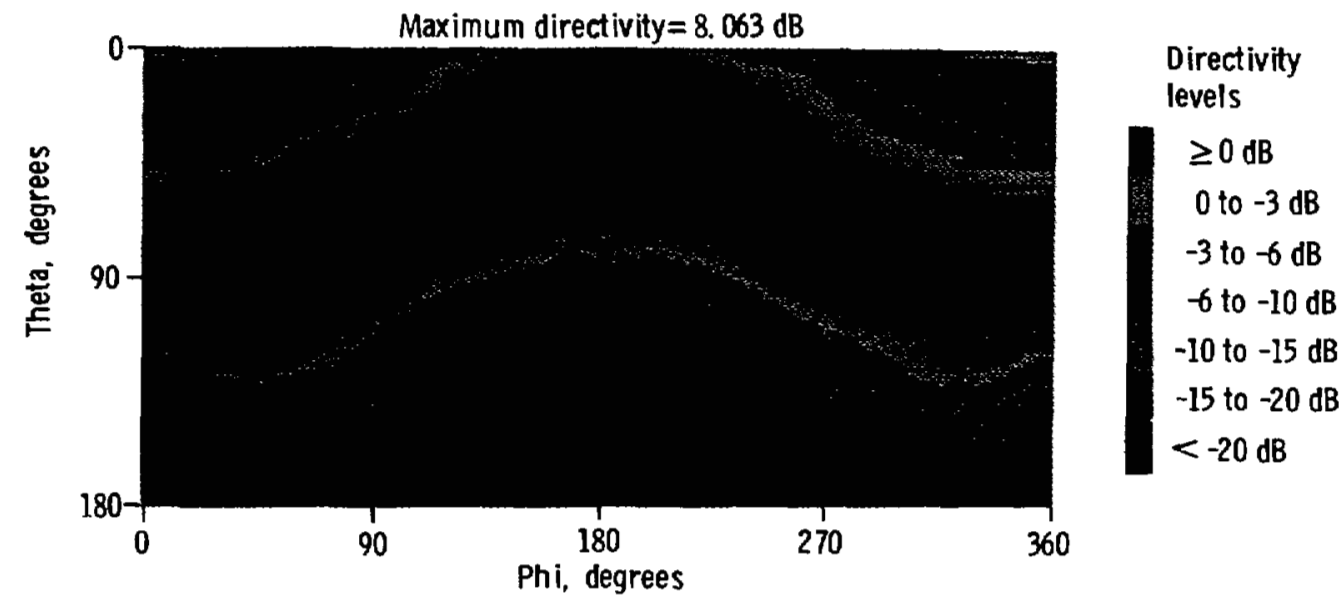

(b) Measured volumetric directive gain pattern of a monopole at station 220

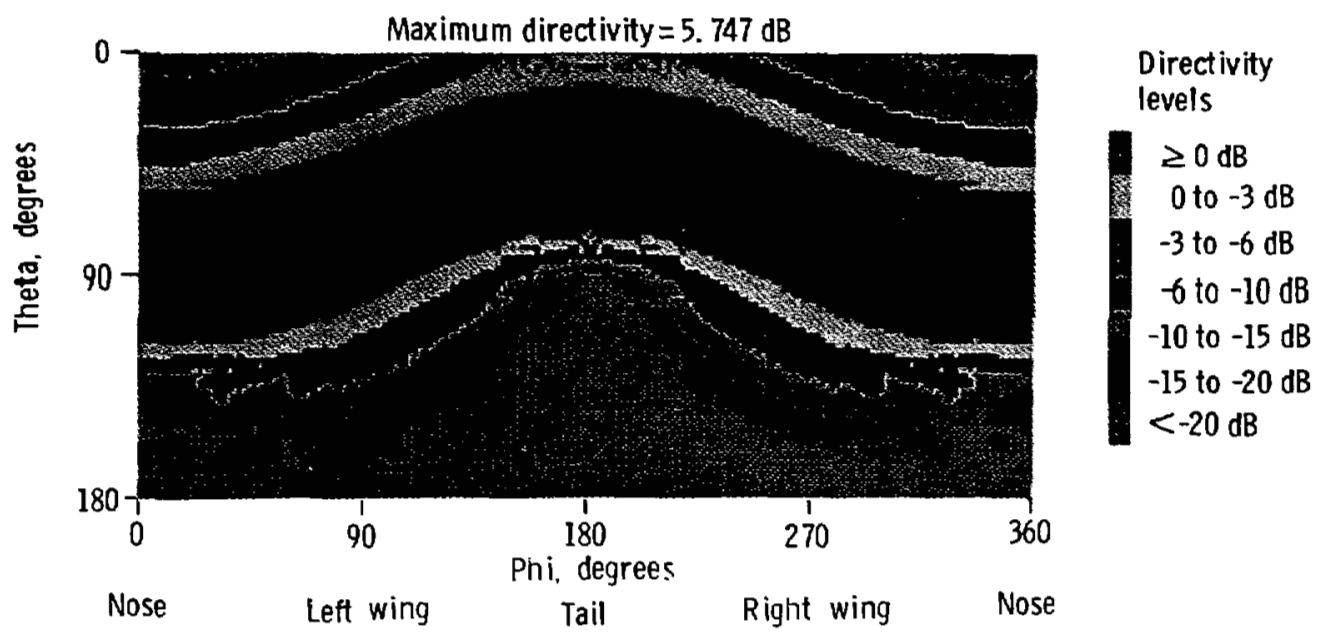

Nose location - Theta $=90^{\circ}$. Phi $=0^{\circ}$

(c) Calculated volumetric directive gain pattern of a monopole at station 220

Fig. 8. Scale model of Boeing 737 and volumetric gain patterns. 


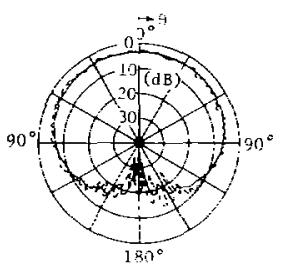

- CALCLLLATEO
-- MEASUIED $3^{V}$

(a)

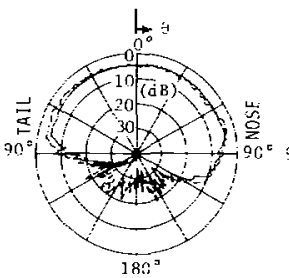

$180^{3}$

- CALCULATEO
--- MEASURES $8 Y$ LINDBERG

(c)

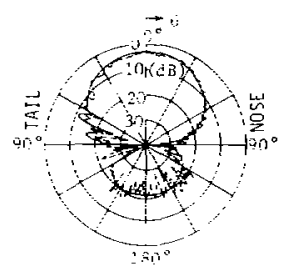

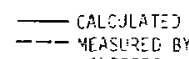
-INTSERG

(t)

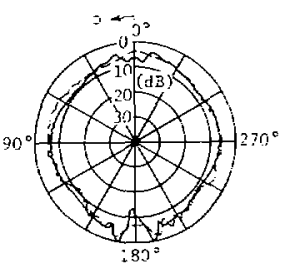

- CAL CULATEE MEASUAED BY
LIVDBERT.

(e)

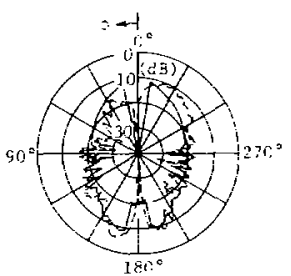

— OALCULATEL

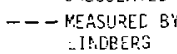

if)
Fig. 9. Radiation patterns of Lindberg crossed-siot antenna mounted at Station 470 on KC-135 aircraft. (a) Roll plane pattern $\left(E_{\theta}\right)$.

(b) Roll plane pattern $\left(E_{\phi}\right)$. (c) Elevation plane pattern $\left(E_{\theta}\right)$.

(d) Elevation plane pattern $\left(E_{\dot{\phi}}\right)$. (e) Azimuth plane pattern $\left(E_{\theta}\right)$.

(f) Azimuth plane pattern $\left(E_{\phi}\right)$.

There are two additional features of this anlaysis, useful to the antenna designer, which have not been discussed. First, this solution provides phase data with no additional effort; yet, accurate phase data generated using a scale-model measurement approach is very difficult. Additionally, this solution is based on the radiation patterns of the three infinitesimal antennas (monopole, axial and circumferential slots). As a result, one can analyze an arbitrary fuselage-mounted antenna simply by performing a numerical integration over the equivalent aperture currents. In a mathematical sense, this far field pattern solution provides a high-frequency asymptotic Green's function for fuselage-mounted airborne antennas. This point has been illustrated in the pattern results presented in this paper in terms of the $K_{a}$-band waveguide and Lindberg antennas. In a similar manner, this solution can be applied to analyze the radiation patterns of arrays using the superposition principle.

\section{REFERENCES}

[1] R. G. Kouyoumjian and P. Pathak, "A uniform geometrical theory of diffraction for an edge of a perfectly conducting surface," in Proc. IEEE, vol. 62, no. 11, pp. 1448-1461, Nov. 1974.

[2] P. Pathak and R. G. Kouyoumjian, "An analysis of the radiation from apertures in curved surfaces by the geometrical theory of diffraction," in Proc. IEEE, vol. 62, no. 11, pp. 1438-1447, Nov. 1974.

[3] W. D. Burnside, R. J. Marhefka, and C. L. Yu, "Roll plane analysis of on-aircraft antennas," IEEE Trans. Antennas Propagat. vol. AP-21, pp. 780-786, Nov. 1973.

[4] W. D. Burnside, M. C. Gilreath, R. J. Marhefka, and C. L. Yu, "A study of KC-135 aircraft antenna patterns," IEEE Trans. Antennas Propagat., vol. AP-23, no. 3, pp. 309-316, May 1975.

[5] W. D. Burnside, "Analysis of on-aircraft antenna patterns," Report 3390-1, Aug. 1972, The Ohio State University ElectroScience Lab., Dep. Electrical Engineering; prepared under Contract N62269-72-C-0354 for Naval Air Development Center.

[6] C. L. Yu and W. D. Burnside, "Volumetric pattern analysis of fuselage-mounted airborne antennas," Report 2902-24, Apr. 1976, The Ohio State University ElectroScience Laboratory, Dep. Electrical Engineering; prepared under Grant Number 36-008-138 for National Aeronautics and Space Administration.

[7] M. C. Gilreath, "Airborne antenna polarization study for the microwave landing system," NASA TM X-73952, July 1976, National Aeronautics and Space Administration, Langley Research Center.

[8] C. A. Lindberg, "A shallow-cavity UHF crossed-slot antenna," IEEE Trans. Antennas Propagat., vol. AP-17, pp. 558-563, Sept. 1969. 